\title{
Characterizing the many-body localization transition by the dynamics of diagonal entropy
}

\author{
Zheng-Hang Sun $\odot,{ }^{1,2}$ Jian Cui $\odot,,^{3, *}$ and Heng Fan ${ }^{1,2,4, \dagger}$ \\ ${ }^{1}$ Institute of Physics, Chinese Academy of Sciences, Beijing 100190, China \\ ${ }^{2}$ School of Physical Sciences, University of Chinese Academy of Sciences, Beijing 100190, China \\ ${ }^{3}$ Department of Physics, Key Laboratory of Micro-Nano Measurement-Manipulation and Physics (Ministry of Education), \\ Beihang University, Beijing 100191, China \\ ${ }^{4}$ CAS Central of Excellence in Topological Quantum Computation, Beijing 100190, China
}

(Received 5 October 2019; revised manuscript received 14 January 2020; accepted 15 January 2020; published 18 February 2020)

\begin{abstract}
Based on the dynamics of diagonal entropy (DE), we provide a nonequilibrium method to study the properties of many-body localization (MBL) transition including the critical point and the universality class. By systematically studying the dynamical behaviors of DE in the fully explored Heisenberg spin chain with quasiperiodic field, we demonstrate the DE method can efficiently detect the transition point $W_{c}$ between the thermal and MBL phase. We further use the method to study the MBL transition in the isotropic $X X$-ladder model, showing $W_{c} \sim 8.05$. We also widely explore the $X X$-ladder model with various parameters. Our results indicate that the MBL transition in the Heisenberg model and the $X X$-ladder model belong to distinct universality classes according to the obvious difference between the scaling exponents. These results can be tested in ongoing quantum simulation experiments with larger qubit numbers, since the diagonal elements of the density matrix directly yielding the DE can be easily obtained by repeatedly running single-shot measurements.
\end{abstract}

DOI: 10.1103/PhysRevResearch.2.013163

\section{INTRODUCTION}

Whether a strongly correlated many-body system thermalizes over time is an intriguing question. Many-body localization (MBL) is a prominent example for the failure of thermalization in interacting systems with strong random or quasiperiodic potential field at nonzero temperatures [1-4]. Recent developments of quantum simulation based on ultracold atoms [5-9], trapped ions [10], and superconduction circuits [11-14] with small coupling to thermal environment pave the way for studying MBL in large-scale systems beyond classical exact diagonalization calculations. Several characteristic dynamical properties of MBL, such as the logarithmic spreading of entanglement entropy (EE) [11,15-20] and quantum Fisher information [10,21,22], and the power-law decay of imbalance [5-8,23-26], are observed.

Since the nonequilibrium properties of quantum systems are naturally available in quantum simulations, considerable attention has been attracted by the intense debate of MBL from the dynamical perspectives, involving the many-body mobility edge [27,28] and MBL transition [29]. Many efforts have been made to find alternative probes of MBL other than $\mathrm{EE}$, because the measurement of $\mathrm{EE}$ requiring quantum

\footnotetext{
*jianCui@buaa.edu.cn

†hfan@iphy.ac.cn
}

Published by the American Physical Society under the terms of the Creative Commons Attribution 4.0 International license. Further distribution of this work must maintain attribution to the author(s) and the published article's title, journal citation, and DOI. state tomography remains a challenge for many qubits systems [11]. Along this direction it has been shown that the dynamical behavior of diagonal entropy (DE), i.e., the Shannon information entropy, also exhibits slow growth in MBL systems [30]. More appealingly, only the diagonal part of the density matrix is required for calculating the DE. Thus, DE can be obtained via repeatedly running single-shot measurements without rotating the measurement basis, which is more efficient than quantum state tomography.

Recently, the transition between the thermal and MBL phase has been a key issue. Notably, the critical disorder strength as well as the critical scaling exponents are widely explored numerically using exact diagonalization method for the system with size up to 22 qubits in cooperation with finite-size critical scaling collapse [31-38]. In addition, the phenomenological renormalization group approaches to the MBL transition also make great progress [39-42]. Although the experimentally feasible quantity DE can identify the MBL by its slow growth, how to characterize the MBL transition and related critical properties via the $\mathrm{DE}$, especially via its nonequilibrium behavior, remains an intriguing open problem.

In this work, we focus on the DE for the whole system of $N$ qubits, namely,

$$
S\left(\rho^{\text {diag }}\right)=-\operatorname{Tr}\left(\rho^{\text {diag }} \log _{2} \rho^{\text {diag }}\right),
$$

where $\rho^{\text {diag }}$ denotes the matrix obtained from the pure state density operator $\rho=\left|\psi_{N}(t)\right\rangle\left\langle\psi_{N}(t)\right|$ by deleting its offdiagonal elements. DE is a genuine measure of quantum coherence for pure states $[43,44]$, which is a basis-dependent quantity. Taking the experimental feasibility into consideration, we choose the Fock basis $\left|\sigma_{1}, \sigma_{2}, \ldots, \sigma_{N}\right\rangle$ (for $N$-qubit system) with $\sigma_{i}=0$ or 1 representing the spin- $z$ component 


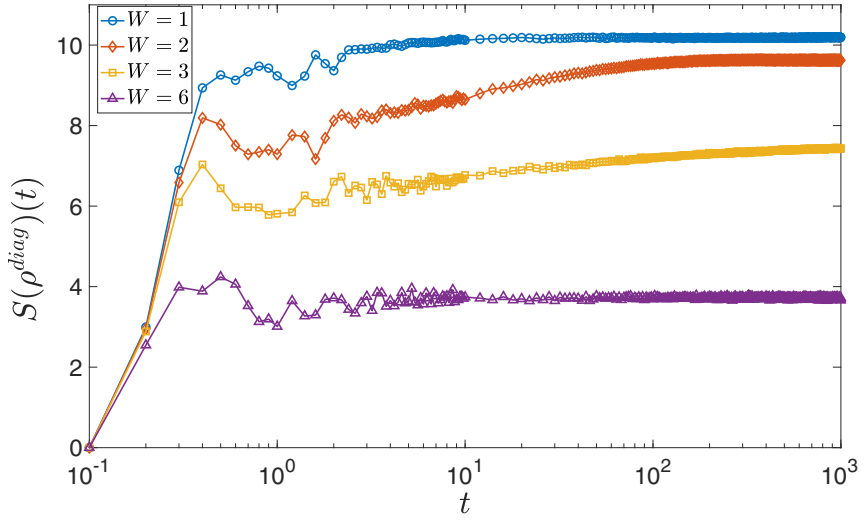

FIG. 1. Semilogarithmic plot of DE $S\left(\rho^{\mathrm{diag}}\right)$ for Heisenberg model $H_{1}$ with $N=14$ and different $W$ as a function of time $t$.

$-1 / 2$ and $+1 / 2$, respectively, because it is naturally the basis of the measurement in quantum simulation experiments $[10,11,13]$. We mainly focus on the time evolution of DE since the quenched scenarios are used in experiments.

This paper is organized in what follows. In Sec. II, we calculate the dynamics of DE in the Heisenberg spin- $1 / 2$ chain with quasiperiodic external field observing the slow evolution of DE when the quasiperiodic potential is strong, followed by a finite-size scaling analyze of the dynamical DE to identify the critical strength as well as the corresponding critical scaling exponents. In Sec. III, using the dynamics of DE we study the MBL transition, involving the transition points and scaling exponents, in $X X$-ladder model with quasiperiodic potential, which in principle can be realized in cold atoms $[45,46]$ and superconducting circuits [47]. Discussions and conclusions are provided in Sec. IV.

\section{HEISENBERG SPIN-1/2 CHAIN}

The Hamiltonian of Heisenberg spin-1/2 chain with a quasiperiodic potential reads

$$
H_{1}=\sum_{i=1}^{N-1} \vec{\sigma}_{i} \cdot \vec{\sigma}_{i+1}+W \sum_{i=1}^{N} \cos (2 \pi c i+\phi) \sigma_{i}^{z},
$$

where $\vec{\sigma}_{i}=\left(\sigma_{i}^{x}, \sigma_{i}^{y}, \sigma_{i}^{z}\right)$ are the Pauli operators for site $i$. The nearest coupling strength is used as the unit of energy and that of inverse time for this model. The strength of quasiperiodic potential is represented as $W$, and $c=\sqrt{2}$ is an irrational number. $\phi$ denotes a global random phase for all $i$ which creates different quasiperiodic potential configurations. The length of Heisenberg spin-1/2 chain is $N$.

We first studied the dynamical behavior of DE by calculating the DE for the total quenched states. Conventionally, we chose the Néel state, i.e., a product state of alternating down and up spins in $z$ direction $|\downarrow \uparrow \downarrow \uparrow \downarrow \uparrow \ldots\rangle$, as the initial state. The number of random phase realization is 200 for $N=16$, 500 for $N=14,1000$ for $N=12$, and 5000 for $N \leqslant 10$. The semilogarithmic plot of the long-time DE dynamics is displayed in Fig. 1. For weak quasiperiodic potential $(W=1)$, the DE firstly experiences a fast growth, and then saturate to a converged value. For a stronger $W$, we observe the slower evolution of DE. Overall, the qualitative behaviors of DE
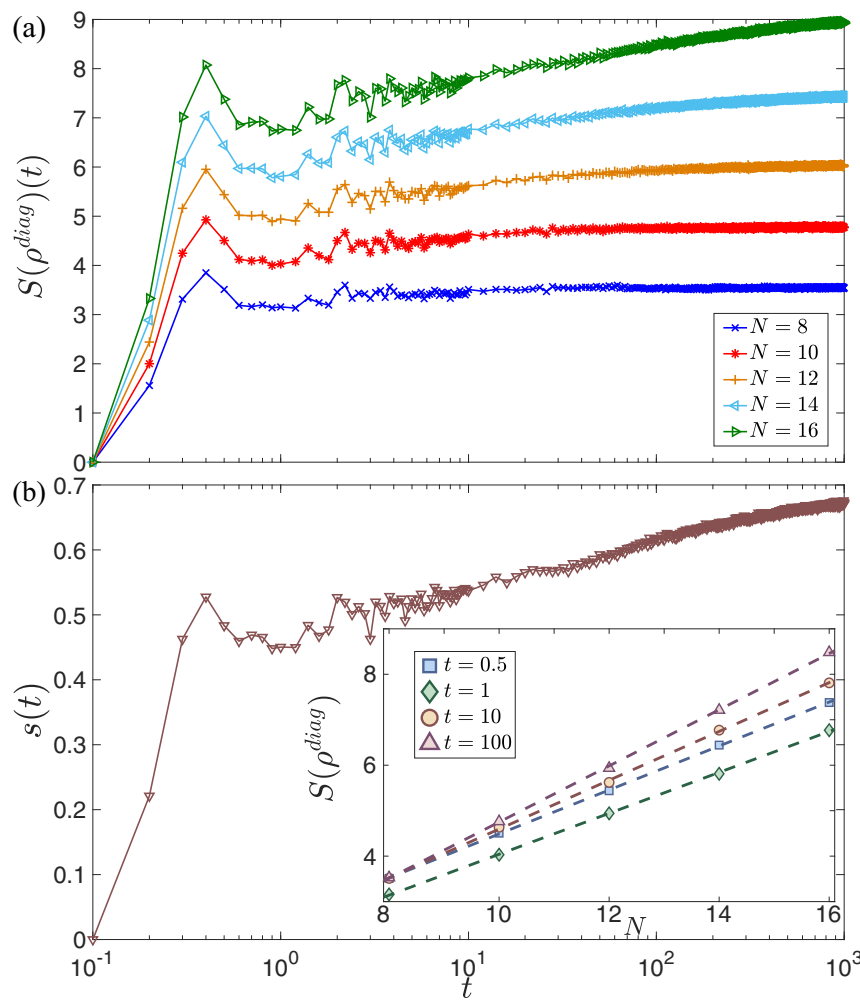

FIG. 2. (a) Semilogarithmic plot of DE $S\left(\rho^{\text {diag }}\right)$ as a function of time $t$ in $H_{1}$ with $W=3$ and different length of the spin chain $N$. (b) The DE density $s$ as a function of time $t$ in $H_{1}$ with $W=3$ and different length of the spin chain $N$. The inset shows the relation between the value of $\mathrm{DE}$ and $N$ at different $t$, and the dashed lines are linear fittings.

are similar to those of EE [17,29,30,36]. Nevertheless, it has been shown in Ref. [36] that the EE has a power-law growth in the delocalized side with a relatively long time interval $t \in[1,10]$, while the time interval of initially rapid growth of DE is shorter $(t<1)$ and less distinguishable.

Moreover, as shown in Fig. 2, there is a volume law of DE in terms of qubit numbers $S\left(\rho^{\text {diag }}\right) \propto s N$, where $s$ denotes the DE density. The volume law is satisfied for the whole duration. This is different from half-chain EE, whose value is independent of $N$ for a wide time interval [36]. The volume effect of DE can be explained by quantum coherence distribution. Generally, the quantum coherence of relative entropy is $\mathcal{C}(\rho)=S\left(\rho^{\mathrm{diag}}\right)-S(\rho)$ with $S(\rho)=-\operatorname{Tr}\left(\rho \log _{2} \rho\right)$ as the von Neumann entropy. The total quantum coherence $\mathcal{C}(\rho)$ is comprised of local coherence $\mathcal{C}_{L}(\rho)$ and intrinsic coherence $\mathcal{C}_{I}(\rho)$, originating from the coherence on every single spin and the coherence between different subsystems, respectively $[43,48,49]$. Consequently, the local coherence is naturally proportional to the number of spin $N$. Here, since we focus on pure quenched states without partial trace and there is no defined subsystems, the local coherence $\mathcal{C}_{L}(\rho)$ becomes the only contribution of quantum coherence and $S(\rho)=0$, thus

$$
S\left(\rho^{\text {diag }}\right)=\mathcal{C}(\rho)=\mathcal{C}_{L}(\rho) \propto N,
$$

which is consistent with the numerical results shown in the inset of Fig. 2(b). 
The approximately logarithmic growth of $\mathrm{DE}$ has been revealed in the Heisenberg spin- $1 / 2$ chain with random potential [30]. In Fig. 2(b), we observe similar behaviors of DE in the system with quasiperiodic potential. In Appendix A, we compare the power-law fit and logarithmic fit for the data in Fig. 2(b) (also for the data in Fig. 6), showing that the logarithmic fit describes the data only sightly better than the power-law fit. The difference between two fits is not dramatic, and the results do not rule out the possibility that the DE develops slowly with a gentle power-law growth. Furthermore, we study the capability of DE to detect the MBL transition. Previous studies of MBL transition were mainly restricted to the properties of the eigenstates for the Hamiltonian at arbitrary energy density [31-37,39-42]. However, the quenched states rather than the eigenstates can be studied more directly in ongoing experiments [5-8,10-12]. Therefore, we study the DE of quenched states at time $t=300$ and apply data collapse to obtain the critical strength $W_{c}$ of quasiperiodic field and the corresponding scaling exponent $v$. This treatment will be repeated at different times $t$.

The data collapse of half-chain EE $S(\rho)$ is conventionally performed by fitting $S(\rho) / S_{T}(\rho)$ to the form $f\left[N^{1 / v}(W-\right.$ $\left.\left.W_{c}\right)\right]$ [34,36,41], where $S_{T}(\rho)=0.5[N \log (2)-1]$ is the Page value for random pure states [50]. Similarly, we can perform the collapse of $S\left(\rho^{\text {diag }}\right) / S_{T}\left(\rho^{\text {diag }}\right)$ to the form $f\left[N^{1 / \nu}(W-\right.$ $\left.W_{c}\right)$ ]. The value of maximum $\mathrm{DE}$ for random pure states is $[30,51,52]$

$$
S_{T}\left(\rho^{\text {diag }}\right) \simeq \log (0.48)+N \log (2) .
$$

In Fig. 3, we show that the best collapsing effect for $t=300$ can be given by $W_{c} \sim 5.5$ and $v \sim 0.38$. We then demonstrate the $W_{c}$ and $v$ obtained from the data collapse at different times in Fig. 4. One can see that the exponent $v$ saturates a stable value for $t \geqslant 200$. The saturated behavior of $v$ can be regarded as a characteristic of the proper time after which one can extract the critical disorder strength from the DE of quenched states. The average value of $W_{c}$ and $v$ for $t \geqslant 200$ is highlighted by the dashed lines in Fig. 4, showing the most appropriate estimation of the transition point is $5.62 \pm 0.35$. In Ref. [36], the MBL transition in the Hamiltonian Eq. (2) is also studied by calculating the entanglement of highly excited eigenstates and the quench dynamics of entanglement entropy and imbalance. The properties of eigenstates only give the lower bound of critical point $W_{c} \sim 3.7-3.9$, while the quench dynamics shows that the signatures of thermalization and MBL appear when $W \sim 4$ and $W \sim 6$, respectively, indicating that the range of critical strength is $W_{c} \in[4,6]$ (note a factor 2 applies as a result of a different definition of the spin matrices). The currently obtained critical disorder strength $W_{c}=5.62 \pm 0.35$ using the data collapse falls in the interval of $W_{c} \in[4,6]$ obtained from Ref. [36].

We also realize that the scaling exponent $v \sim 2.6$ of $\mathrm{DE}$ is different from that of EE in Ref. [36] $v \sim 1$.1. Actually, the scaling exponents obtained via the data collapse of different quantities may have divergent values of scaling exponents. For example, in Ref. [32], it is found that the data collapse of entanglement entropy and level spacing ratio yield $v=0.801$ and 0.917 , respectively.

We note that the slow growth of DE occurs in the Heisenberg model with $W$ smaller than $W_{c}$; see Fig. 2. This may
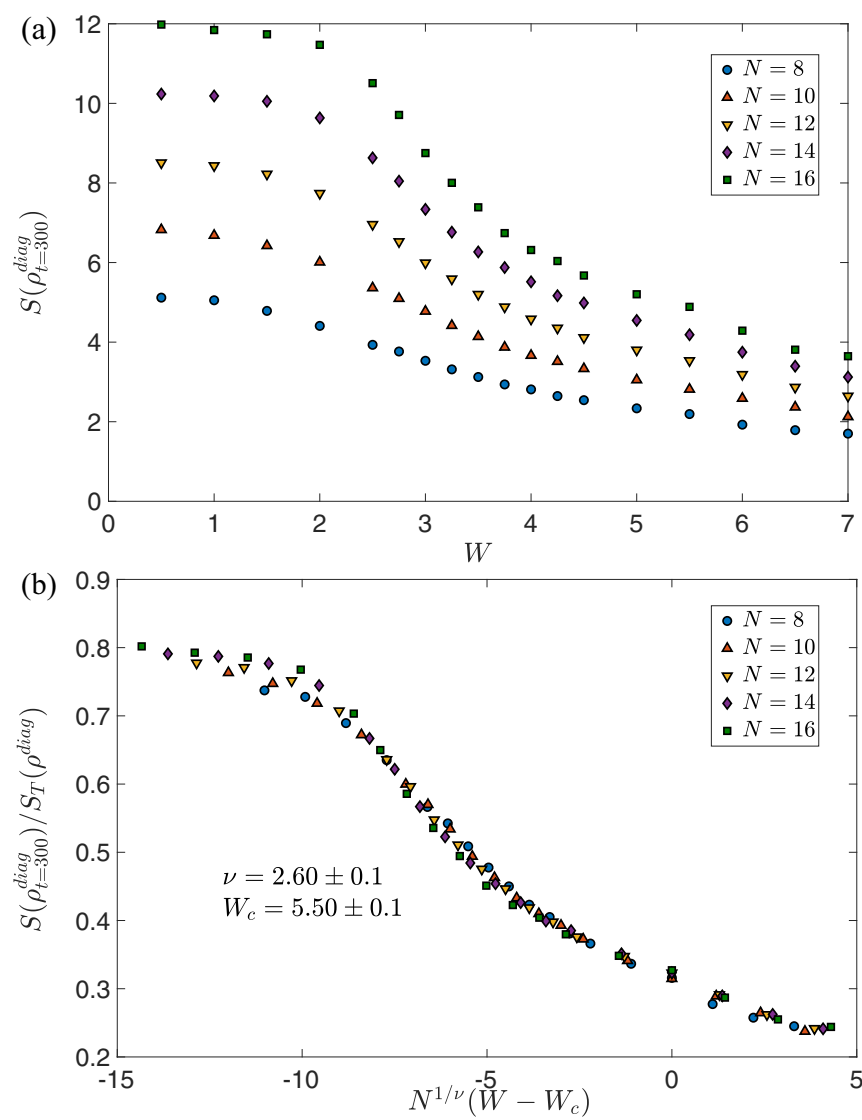

FIG. 3. The value of DE at time $t=300$ as a function of quasiperiodic strength $W$ with different length of the Heisenberg spin chain $N$. (b) Finite-size critical scaling collapse for DE data. The critical quasiperiodic strength $W_{c} \sim 5.4-5.6$ and scaling exponent $v \sim 2.5-2.7$ are used to the most appropriate data collapse.

be induced by the intermediate region where the system experiences a transition between chaos to localization, and the logarithmic growth of EE can also be observed in this region [30]. Nevertheless, the critical point can be accurately obtained by the data collapse of DE.

\section{III. $X X$ SPIN-1/2 LADDER}

\section{A. Hamiltonian}

In the past few years, the quantum criticality [53-55], transport [45,56], and entanglement [57] properties of spin$1 / 2$ Heisenberg model on ladder lattice (Fig. 5),

$$
H_{2}=J_{\|} \sum_{j=1,2} \sum_{i=1}^{M-1} \vec{\sigma}_{j, i} \cdot \vec{\sigma}_{j, i+1}+J_{\perp} \sum_{i=1}^{M} \vec{\sigma}_{1, i} \cdot \vec{\sigma}_{2, i+1},
$$

have been widely investigated. In addition, the MBL transition in the spin-1/2 Heisenberg ladder is explored [58,59]. Other variations on ladder lattice are also studied. For instance, the quasi-many-body-localizations in the spin- $1 / 2$ ladder with only $X Y$ coupling in the $J_{\|}$term and Ising interaction in the $J_{\perp}$ term are revealed [60].

Given the fact that the $X Y$-type coupling can be easier realized on cold atoms [45,46], superconduction circuits $[11,12,47]$, and trapped ions [61] than on Heisenberg 

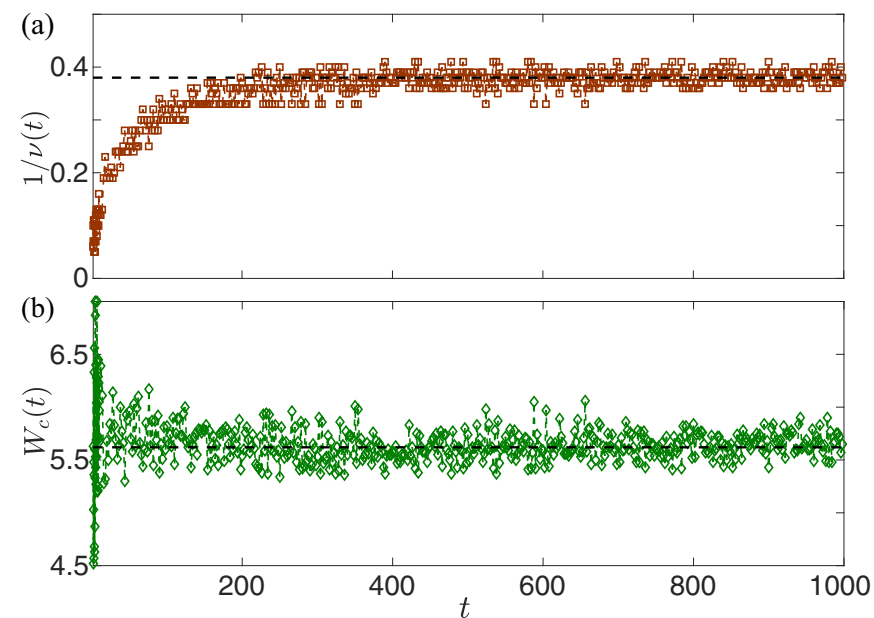

FIG. 4. The $W_{c}$ and $v$ obtained from the data of other time $t$ via the same data collapse method in Fig. 3. The dashed line in (a) and (b) is $1 / v=0.38$ and $W_{c}=5.62$, respectively, obtained from the average of data with $t \geqslant 200$.

interactions, we consider the $X X$-ladder model

$$
\begin{aligned}
& H_{3}=J_{\|} H_{\|}+J_{\perp} H_{\perp}+W H_{z}, \\
& H_{\|}=\sum_{j=1,2} \sum_{i=1}^{M-1}\left(\sigma_{j, i}^{x} \sigma_{j, i+1}^{x}+\sigma_{j, i}^{y} \sigma_{j, i+1}^{y}\right), \\
& H_{\perp}=\sum_{i=1}^{M}\left(\sigma_{1, i}^{x} \sigma_{2, i}^{x}+\sigma_{1, i}^{y} \sigma_{2, i}^{y}\right), \\
& H_{z}=\sum_{i=1}^{M} \cos (2 \pi c i+\phi)\left(\sigma_{1, i}^{z}+\sigma_{2, i}^{z}\right),
\end{aligned}
$$

where $M=N / 2$ is the length of the spin ladder composed of $N$ qubits, $W$ is the strength of quasiperiodic potential, and $J_{\|}$ and $J_{\perp}$ refer to the intrachain and rung hopping coefficients, respectively. The ratio of interactions is denoted as $\alpha=J_{\perp} / J_{\|}$. With $\alpha=1$, i.e., $J_{\perp}=J_{\|}$, the $X X$ spin ladder is isotropic. A schematic representation of the Hamiltonian Eq. (6) and the initial state for the time evolution are shown in Fig. 5.

It has been analytically shown that there are interactions between the spinless fermions after Jordan-Wigner transformation in $H_{3}$ [62] (also see Appendix B). We emphasize that the interactions in Hamiltonian Eqs. (2) and (6) inducing

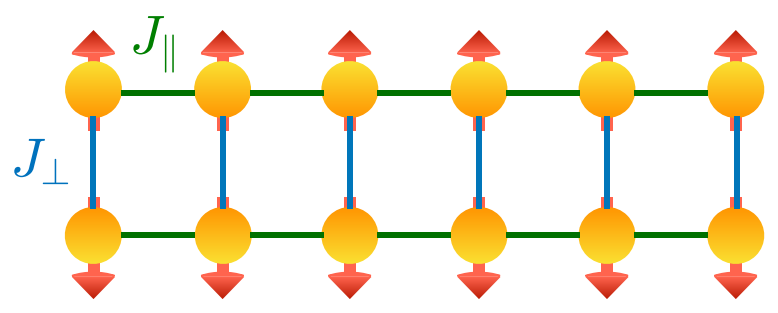

FIG. 5. Schematic of the $X X$-ladder model. The $X Y$-type intrachain and rung hopping interactions are marked by the green and blue lines with the strength $J_{\|}$and $J_{\perp}$ respectively. The up and down arrows at each spins refer to the state $|\uparrow\rangle$ and $|\downarrow\rangle$ as the eigenstates of $\sigma^{z}$ with eigenvalue 1 and -1 . The initial state is the product state.

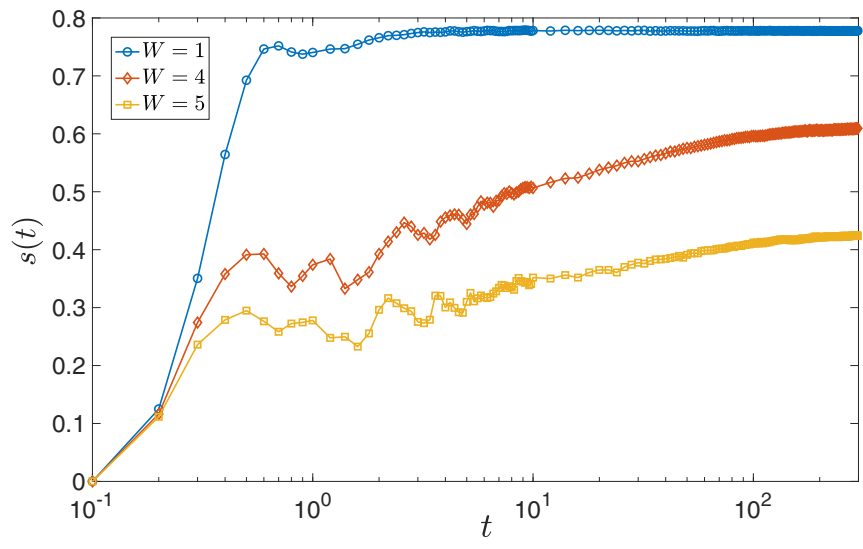

FIG. 6. The time evolution of DE density $s$ for the $X X$-ladder model with $J_{\|}=J_{\perp}=1$ different values of $W$.

MBL are different. In the Heisenberg spin chain Eq. (2), the interactions $\sigma_{i}^{z} \sigma_{i+1}^{z}$ (denoted as Heisenberg coupling) lead to $c_{i}^{\dagger} c_{i} c_{i+1}^{\dagger} c_{i+1}$ with $c_{i}^{\dagger}$ and $c_{i}$ the fermionic creation and annihilation operators. However, in the $X X$-ladder model, the interactions have roots in the string operator. Indeed, when $W=J_{\perp}=0$, the Hamiltonian Eq. (6) degenerates into two independent $X Y$ spin chains whose integrability is tightly related to the fact that the nearest interaction of string operator only results in a quadratic form of the fermion operators [63], which is violated by the $X X$-ladder model in general.

It has been shown that the average ratio of level spacing $\bar{r} \sim 0.53$, as an evidence of thermalization, for the $X X$-ladder model with $\alpha \sim 1$ and weak quasiperiodic potential strength, while $\bar{r}$ approaches to 0.39 when $\alpha \rightarrow 0$ [45]. Hence, $\alpha=1$ is an appropriate case for studying MBL as a breakdown of thermalization. In the following part, by calculating the dynamics of DE, we study the MBL transition in the $X X$-ladder model with $\alpha=1$ and 0.7 , along with a discussion of the universality classes of the corresponding MBL transitions. Additionally, in Appendix C, we study the model with $\alpha=0.1$ and present the signatures of nonergodicity and Anderson localization, in comparison with the thermalization (ergodicity) and MBL, via the time evolution of DE. The dependence of DE dynamics and initial states is also explored in Appendix C.

\section{B. Results of $\alpha=1$}

We firstly consider the $X X$-ladder with $\alpha=1$ and $J_{\|}=1$. In Fig. 6, we show fast saturation and slow growth for $H_{3}$ with weak $(W=1)$ and stronger $(W=4,5,8)$ quasiperiodic potential strength, respectively, numerically demonstrating the occurrence of thermalization and MBL in this model.

Furthermore, we use the same method as applied in the Heisenberg spin chain to extract the critical strength of quasiperiodic potential $W_{c}$ from the dynamics of DE employing data collapse. In Fig. 7, we depict the DE of quenched states at $t=300$ as a function of $W$ and the results of the most appropriate data collapse yielding $W_{c} \sim 8.25$ and $v \sim 1.72$. Considering the time dependence of $W_{c}$ and $v$ shown in Fig. 8, the best estimation of the transition point $W_{c}$ is $8.05 \pm 0.5$ using the DE method, and the scaling exponent is $v \sim 1.70$. 

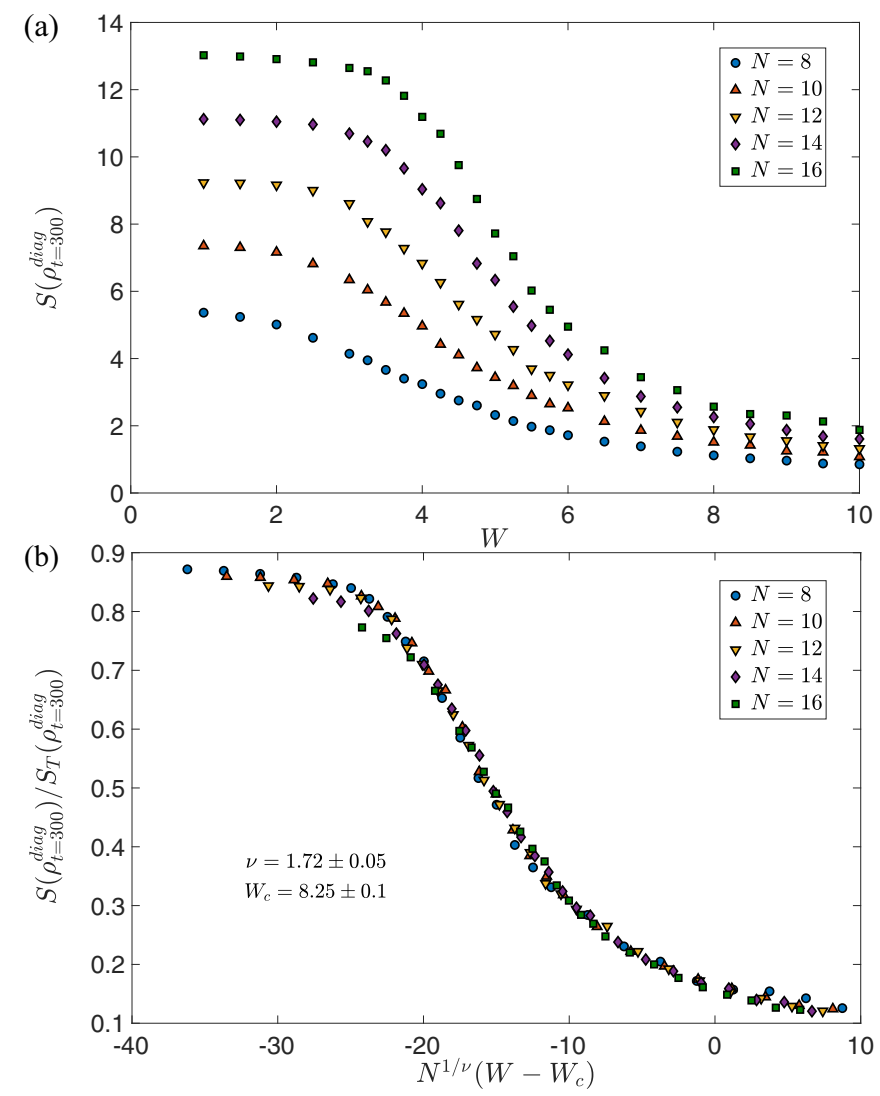

FIG. 7. (a) The value of DE at time $t=300$ as a function of quasiperiodic strength $W$ with different length of $X X$ spin ladder $N / 2$ and $\alpha=1$. (b) Finite-size critical scaling collapse for DE data. The critical quasiperiodic strength $W_{c} \sim 8.15-8.35$ and scaling exponent $v \sim 1.67-1.77$ are used to the most appropriate data collapse.

\section{Results of $\alpha=0.7$}

We then explore the impact of $\alpha$ on the MBL transition in the $X X$-ladder model. Here, we still let $J_{\|}=1$. Before
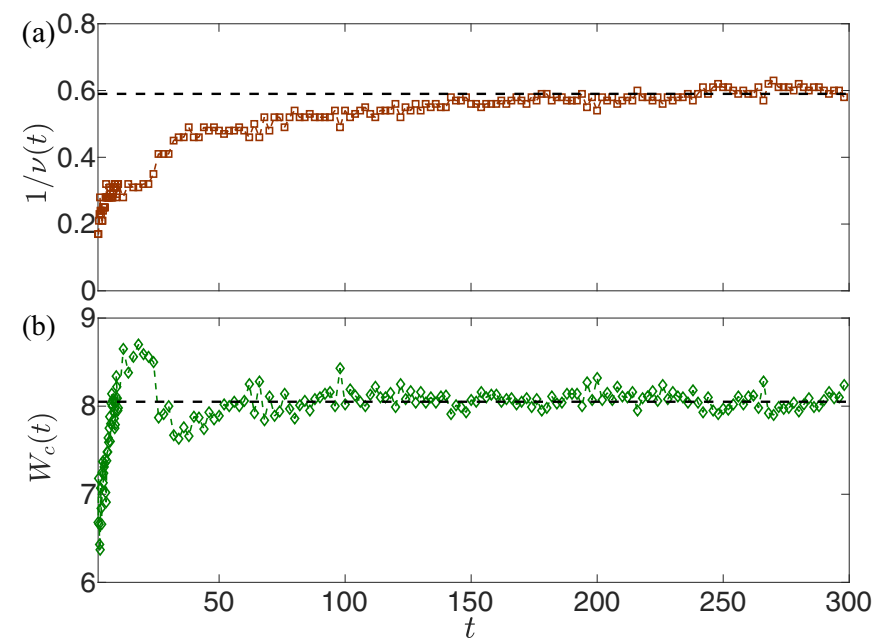

FIG. 8. The $W_{c}$ and $v$ obtained from the data of other time $t$ via the same data collapse method for the $X X$-ladder model with $\alpha=1$. The dashed line in (a) and (b) is $1 / v=0.59$ and $W_{c}=8.05$, respectively, obtained from the average of data with $t \geqslant 150$.

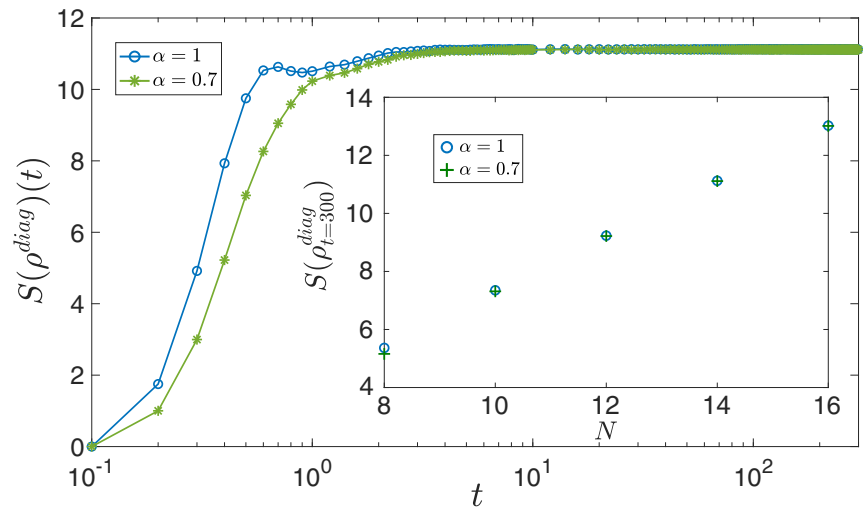

FIG. 9. The time evolution of DE for the $X X$-ladder model with $\alpha=0.7, N=14$ and $W=1$. Inset shows the values of $\mathrm{DE}$ at $t=300$ as a function of system size $N$ with $W=1$.

we study the MBL transition, we should first clarify the occurrence of thermalization in the system with other values of $\alpha$. In Fig. 9, we present the dynamics of DE with a weak strength of quasiperiodic strength $W=1$ for different values of $\alpha=1,0.7$. It is shown that for both $\alpha=1$ and $\alpha=0.7$, the DE ultimately saturates at the same value, which is also demonstrated for different system size $N$, as shown in the inset of Fig. 9. The results in Fig. 9 give a clear signature of the thermalization when $\alpha=0.7$.

Consequently, we can calculate the dynamics of DE in the $X X$-ladder model with $\alpha=0.7$ to explore the dependence of MBL transition on $\alpha$. In Fig. 10, we depict the DE of quenched states at $t=300$ and the results of the most appropriate data collapse with obtained transition point $W_{c}$ and scaling exponent $v$. The time dependence of the $W_{c}$ and $v$ is shown in Fig. 11. Based on the above results, for the $X X$-ladder model with $\alpha=0.7$, the transition point can be estimated as $W_{c} \sim$ 7.57 which is smaller than that for $\alpha=1$. The breakdown of thermalization requires weaker quasiperiodic potential for the model with $\alpha=0.7$.

\section{The scaling exponents}

The value of scaling exponent can provide valuable insights to the universality classes for the MBL transitions. For instance, recent works show that the randomness-induced and quasiperiodic-induced MBL belong to two distinct universality classes according to the significant difference between the scaling exponents obtained from the data of $\mathrm{EE}[34,39]$. Thus, to study the universality classes of MBL transitions, one can focus on the difference of scaling exponents obtained from the data of same quantity in different systems. We notice that all the obtained scaling exponents in this work respect the HarrisLuck bound $v>1 / d$ with $d=1$ for the one-dimensional quasiperiodic systems [64-66]. The satisfaction of the HarrisLuck bound indicates that the scaling ansatz $S(\rho) / S_{T}(\rho) \sim$ $f\left[N^{1 / v}\left(W-W_{c}\right)\right]$ is appropriate, and the scaling exponent $v$ can be employed to classify the universality classes of the MBL transitions.

Our results show that there is an obvious difference between the scaling exponent $v$ obtained from the data of DE for the MBL transition in the Heisenberg spin chain (see Fig. 4(a), 

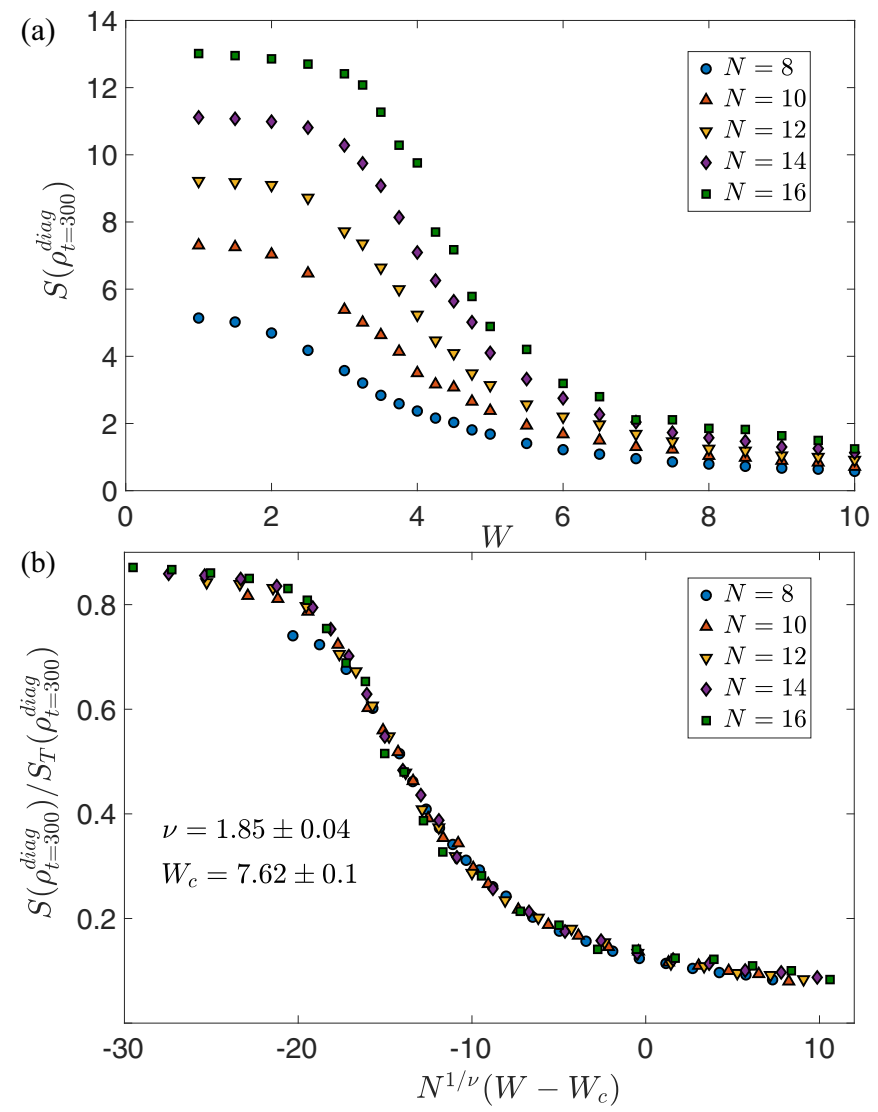

FIG. 10. (a) The value of DE at time $t=300$ as a function of quasiperiodic strength $W$ with different length of $X X$ spin ladder $N / 2$ and $\alpha=0.7$. (b) Finite-size critical scaling collapse for DE data. The critical quasiperiodic strength $W_{c} \sim 7.52-7.72$ and scaling exponent $v \sim 1.81-1.89$ are used to the most appropriate data collapse.

$v \sim 2.63$ ) and that in the $X X$-ladder model with $\alpha=1$ (see Fig. 8(a), $v \sim 1.70$ ). The above difference of $v$ suggests that, besides the random or quasiperiodic potential, the origin of interaction should also be considered for the classifications of MBL transitions since the former is from the Heisenberg coupling, while the latter is due to the nonlocal string operator.

In addition, the comparison between the results in Figs. 8(a) and 11(a) indicates that for the $X X$-ladder model, the scaling exponent $v$ is dependent on $\alpha$, i.e., $v \sim 1.89$ and $v \sim 1.70$ when $\alpha=0.7$ and 1 , respectively. When changing $\alpha$ from 1 to 0 , one can presumably predict that there is a transition between MBL and Anderson localization (AL) for the system with a strong quasiperiodic strength. It has been shown that the scaling exponent for $\mathrm{AL}$ is different from that of MBL [34,39]. Thus, the dependence of $v$ on $\alpha$ may be regarded as an influence for the transition between MBL and AL.

\section{SUMMARY AND DISCUSSION}

We have studied the dynamical behaviors of DE in interacting spin models with quasiperiodic field. Similar to EE, the slow growth of DE is also observed. More importantly, the critical field strength $W_{c}$ of MBL transition can be obtained from the dynamics of DE via data collapse as an analysis of
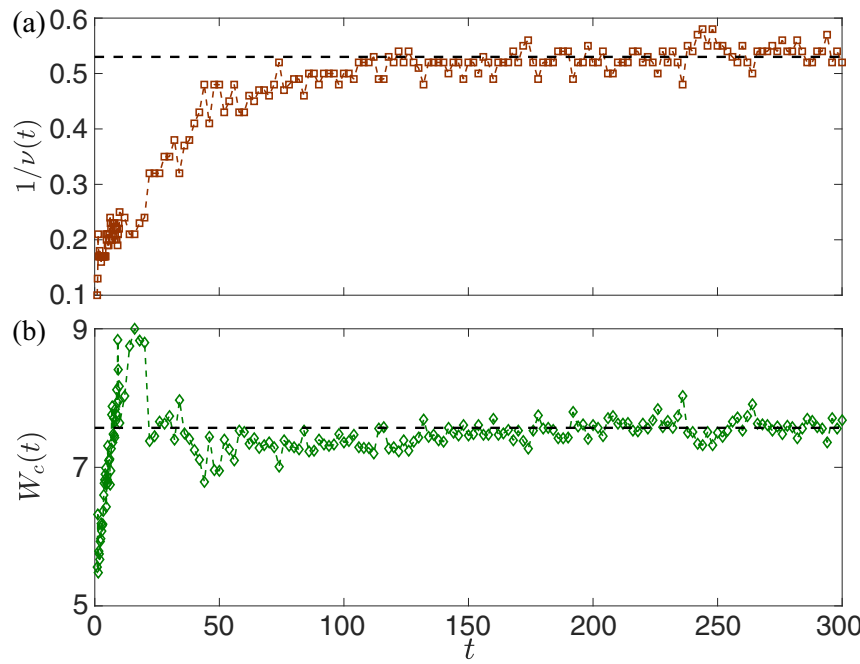

FIG. 11. The $W_{c}$ and $v$ obtained from the data of other time $t$ via the same data collapse method for the $X X$ spin ladder with $\alpha=0.7$. The dashed line in (a) and (b) is $1 / v=0.53$ and $W_{c}=7.57$, respectively, obtained from the average of data with $t \geqslant 150$.

finite size effect. After demonstrating that in the Heisenberg spin chain, $W_{c} \sim 5.62$ [36], we use the same DE method to study the MBL transition in the $X X$-ladder model, which has not been fully explored. For the first time, we show that $W_{c} \sim 8.05$ for the spin ladder. We also study the spin ladder with other parameters, showing the impact of the interaction ratio $\alpha$ on the MBL transition, and the signature of $\mathrm{AL}$ and nonergodicity characterized by the dynamics of DE.

In our work, all studied MBL is induced by quasiperiodic potential. However, the difference between $v$ for the MBL transition in the systems studied here is quite distinguishable, indicating that the randomness-induced and quasiperiodicinduced MBL may not cover all possible universality classes [34]. Nonetheless, the results of our work are obtained by exact diagnolization, which is worthy of further investigation by using renormalization group approaches or calculating other quantities.

We believe that our results can be directly verified in various quantum simulators, especially in the superconducting quantum processor or Rydberg quantum simulators with ladder-type circuit architecture [47,67]. Here, we quantitatively clarify why the measurement of DE is quite efficient in comparison with (half-chain) EE. For a $N$ qubit system, one can measure the diagonal elements of the total system without partial trace to obtain the $\mathrm{DE}$, or measure the reduced density matrix of the subsystem composed of $N / 2$ qubits to obtain the EE. For the former, one can naturally assume that $m \times 2^{N}$ times measurement is required to get enough statistics to obtain the the diagonal elements (usually $m \geqslant 10$ ). In contrast, the quantum state tomography of a reduced density matrix with dimension $2^{N / 2}$ consists of following procedures: (i) Applying a multiqubit rotation pulse $U=\otimes_{i} u_{i}$ where $u_{i}$ is randomly chosen from the set of single-qubit rotation $\{Y / 2, X / 2, I / 2\}$ pulse. Thus, there are $3^{N / 2}$ options of $U$. (ii) After the multiqubit rotation pulse $U$, one has to measure the diagonal elements of the reduced density matrix in the basis, each requiring $m \times 2^{N / 2}$ times measurement. (iii) For different 
$U=\otimes_{i} u_{i}$, one has to repeat (i) and (ii). As a consequence, the total times of measurement for $\mathrm{DE}$ and $\mathrm{EE}$ are $M_{\mathrm{DE}}=$ $m \times 2^{N}$ and $M_{\mathrm{EE}}=m \times 2^{N / 2} \times 3^{N / 2}$, respectively. The ratio is $M_{\mathrm{DE}} / M_{\mathrm{EE}}=(3 / 2)^{N / 2}>1$. Hence, the measurement of DE is more efficient than the EE, especially for multiqubit systems.

Besides, from the numerical and theoretical perspective, this work may shed lights on the further studies of MBL transition in the $X X$-ladder model employing other observables including imbalance or inverse participation ratio, and the behaviors of DE in other systems where MBL occurs, for instance, the systems with a linear potential $[68,69]$. However, this work can also enlighten the characterization of other out-of-equilibrium phenomena such as dynamical phase transitions [70], and information scrambling [71], by DE.

\section{ACKNOWLEDGMENTS}

Z.H.S. acknowledges enlightening discussions with D. N. Sheng, Chen Cheng, Rubem Mondaini, Kai Xu, and Ceren B. Dağ. J.C. was supported by NSFC (Grant No. 11904018). H.F. was supported by NSFC (Grant Nos. 11774406, 11934018), National Key R\&D Program of China (Grants No. 2016YFA0302104 and No. 2016YFA0300600), Strategic Priority Research Program of Chinese Academy of Sciences (Grant No. XDB28000000), and Beijing Academy of Quantum Information (Grant No. Y18G07).

\section{APPENDIX A: LOGARITHMIC VERSUS POWER-LAW FIT}

In this Appendix, we present a comparison of the logarithmic versus power-law fit to the growth of DE. Specifically, we focus on the data of DE density in Fig. 2(b) and in Fig. 6 with $W=4$. We chose an appropriate time interval to avoid the influence of early evolution and final saturation. The results are shown in Fig. 12, indicating that the logarithmic fit describes the numerical data sightly better than the power-law fit.

\section{APPENDIX B: JORDAN-WIGNER TRANSFORMATION FOR THE $X X$-LADDER MODEL}

In this Appendix, we apply the generalized Jordan-Wigner transformation [54,62] to reveal the spinless fermion interactions in the Hamiltonian $H_{4}=H_{\|}+H_{\perp}$ with the $H_{\|}$and $H_{\perp}$ given in Eq. (6). The generalized Jordan-Wigner transformation is described as

$$
\begin{aligned}
& S_{1, l}^{-}=c_{1, l} Q_{1, l}, \\
& S_{2, l}^{-}=c_{2, l} Q_{2, l},
\end{aligned}
$$

where $S_{j, l}^{-}=\left(\sigma_{j, l}^{x}-i \sigma_{j, l}^{y}\right) / 2(j=1,2$ and $l=0,2, \ldots, M-$ 1 with $M$ as the length of ladder), and the string operators read

$$
\begin{aligned}
& Q_{1, l}=\exp \left[i \pi \sum_{k=0}^{l-1}\left(n_{1, k}+n_{2, k}\right)\right], \\
& Q_{2, l}=\exp \left[i \pi\left(\sum_{k=0}^{l} n_{1, k}+\sum_{k=0}^{l-1} n_{2, k}\right)\right],
\end{aligned}
$$

with $n_{j, l}=c_{j, l}^{\dagger} c_{j, l}$ as the spinless fermion number operators for each site. Thus, we have the Hamiltonian $H_{4}$ represented
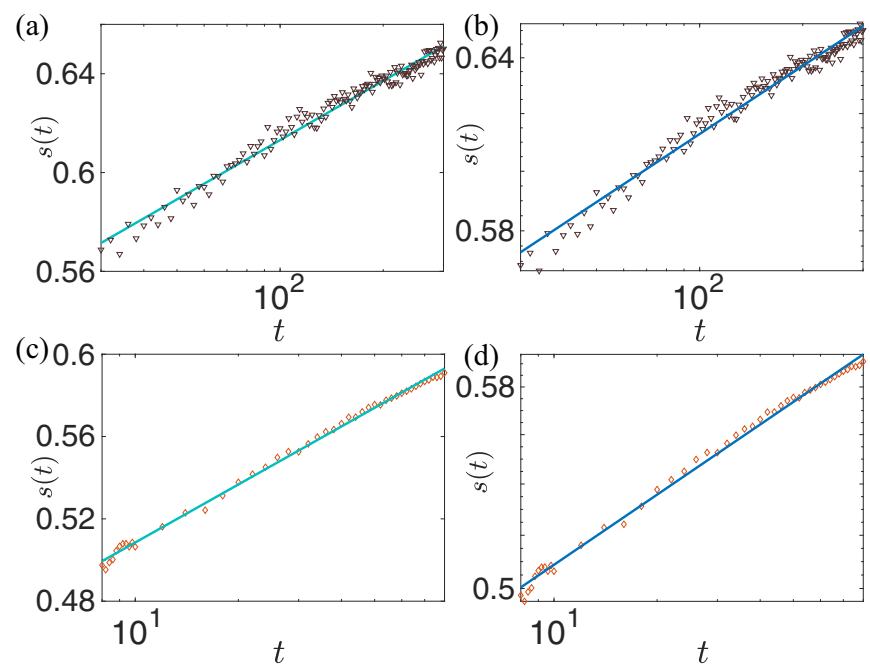

FIG. 12. (a) The data from Fig. 2(b) with the time interval $t \in$ $[30,300]$ on a double-log plot with a power-law fit. (b) The data from Fig. 2(b) with the time interval $t \in[30,300]$ on a logarithmic $x$ axis with a logarithmic fit. The goodness of fitting for the power-law fit is $R^{2}=97.49 \%$ and the fit error (the same definition in Ref. [23]) is 0.00267 . The goodness of fitting for the logarithmic fit is $R^{2}=$ $97.76 \%$ and the fit error is 0.00252 . (c) and (d) is similar to (a) and (b), respectively, but for the data from Fig. 6 with $W=4$ and the time interval $t \in[8,80]$. The goodness of fitting for the power-law fit is $R^{2}=99.53 \%$ and the fit error is 0.00184 . The goodness of fitting for the logarithmic fit is $R^{2}=99.71 \%$ and the fit error is 0.00144 . The lines are fitting functions and the triangle and diamond points are the fitted data from Figs. 2(b) and 6, respectively.

by spinless fermion operators

$$
\begin{aligned}
H_{4}=-2 J_{\|} \sum_{l, \delta} & {\left[c_{1, l} \exp \left(-i \pi \phi_{l, l+\delta}^{(1)}\right) c_{1, l+\delta}^{\dagger}\right.} \\
& \left.+c_{2, l} \exp \left(-i \pi \phi_{l, l+\delta}^{(2)}\right) c_{2, l+\delta}^{\dagger}\right] \\
& -2 J_{\perp} \sum_{l} c_{l, 1} c_{l, 2}^{\dagger},
\end{aligned}
$$

where $\delta$ is the nearest neighbors of a site, and the phase operators refer to $\phi_{l, l+1}^{(1)}=n_{2, l}, \phi_{l, l-1}^{(1)}=-n_{2, l-1}, \phi_{l, l+1}^{(2)}=n_{1, l}$, and $\phi_{l, l-1}^{(1)}=-n_{1, l-1}$. It is obvious that the Hamiltonian $H_{4}$ can not be written as a quadratic form because of the phase operators, and there are interactions that can induce MBL in the system with strong random or quasiperiodic potential field.

\section{APPENDIX C: CHARACTERIZING ERGODIC DYNAMICS AND ANDERSON LOCALIZATION BY DIAGONAL ENTROPY}

In the main text, we study the $\mathrm{DE}$ in the $X X$-ladder model with $\alpha=1$ and 0.7 , showing the distinguishable dynamical behaviors of $\mathrm{DE}$ in the thermal and MBL phase. In this Appendix, we also study the model with $\alpha=0.1$ close to the integrable limit $\alpha \rightarrow 0$, where the Anderson localization (AL) will occur in the presence of a strong quasiperiodic potential. Here, the main interest is in the difference of the dynamical behaviors of DE in the thermal phase and integrable limit, as well as that in the MBL and AL phase. 

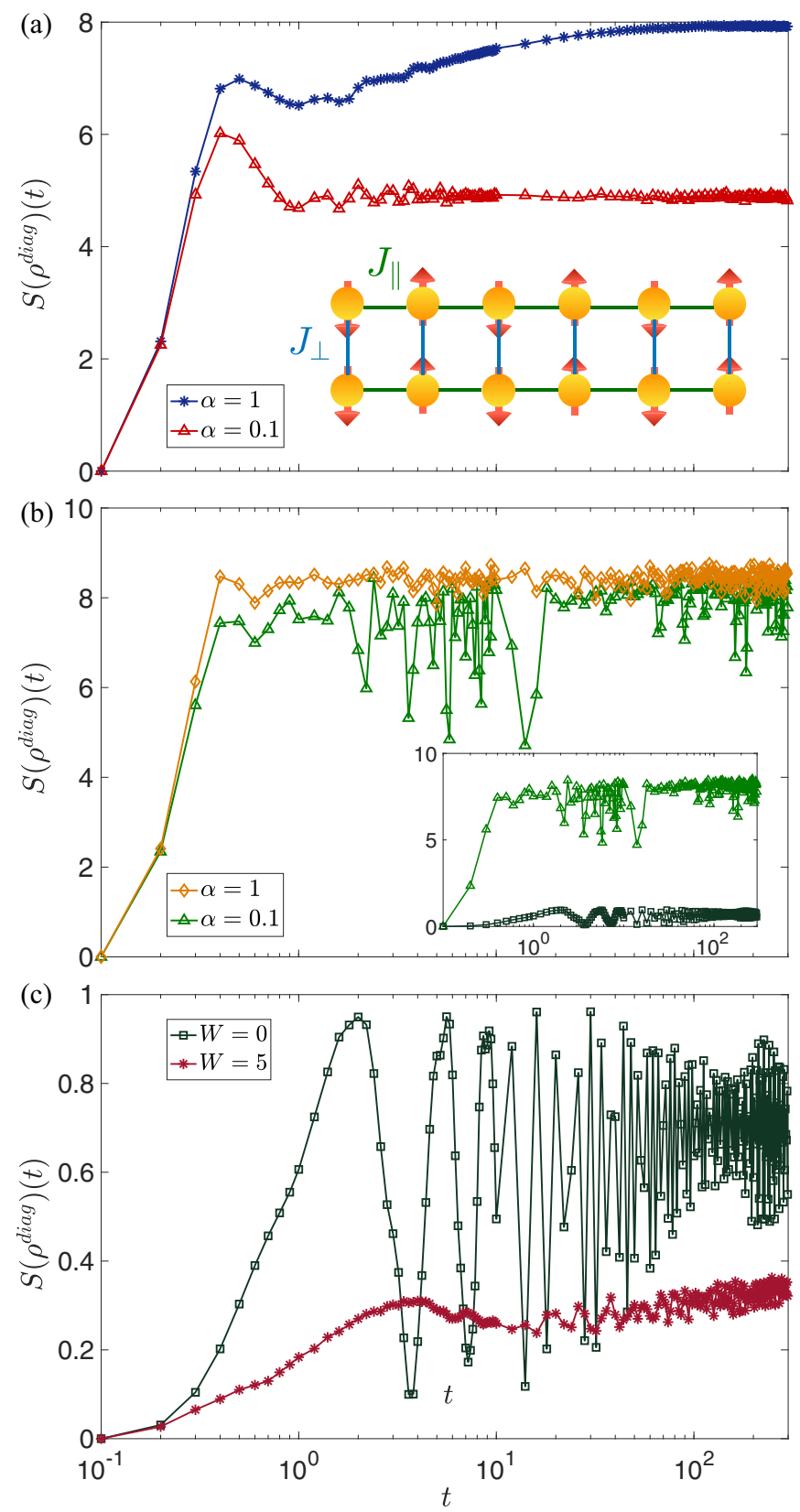

FIG. 13. (a) The dynamics of DE for the $X X$-ladder model with $N=12, \alpha=0.1,1$, and $W=5$. The inset of (a) shows the schematic of the $X X$-ladder model and the chosen initial state. (b) The dynamics of DE for the $X X$-ladder model with $N=12, \alpha=0.1,1$, and $W=0$. The inset of (b) present a comparison between the DE dynamics with the initial state in the inset of Fig. 13(a) (green triangle points) and in Fig. 5 (darker green square points) when $\alpha=0.1$. (c) The dynamics of DE with the initial state in Fig. 5 for the $X X$-ladder model with $\alpha=0.1$ and different $W$.

In Fig. 13(a), we present the dynamics of DE in $H_{3}$ with $N=12, W=5$, and $\alpha=0.1,1$. The initial state is shown in the inset of Fig. 13(a). We observe that there is an obvious logarithmic growth of DE with $\alpha=1$, while the DE saturates to a relatively small value with $\alpha=0.1$, giving a comparison between the MBL and AL. The similar distinctions have also been revealed in the quench dynamics of EE [15]. Without
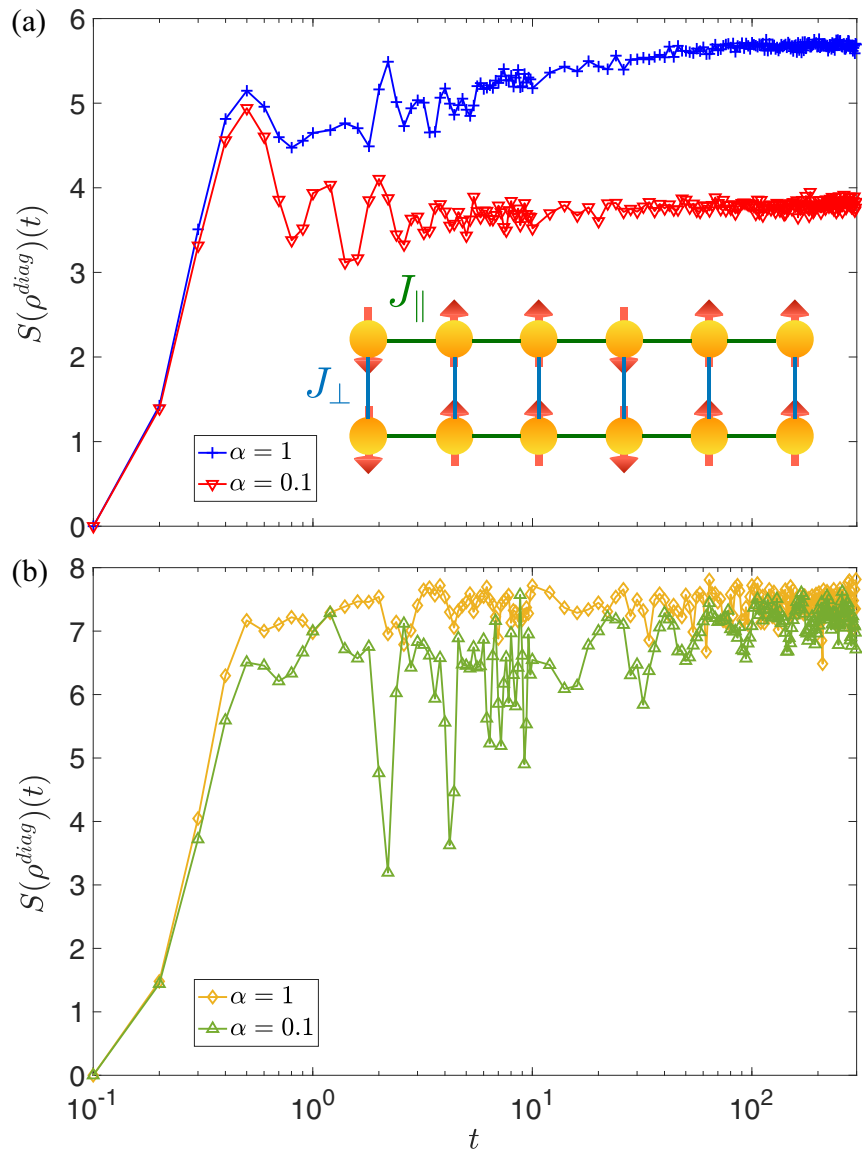

FIG. 14. (a) The dynamics of DE for the $X X$-ladder model with $N=12, \alpha=0.1,1$, and $W=5$. The inset shows the schematic of the $X X$-ladder model and the chosen initial state. (b) The dynamics of DE for the $X X$-ladder model with $N=12, \alpha=0.1,1$, and $W=0$.

quasiperiodic potential, i.e., $W=0$, in Fig. 13(b), we observe dramatic oscillations of DE for $\alpha=0.1$. Instead, for $\alpha=1$, the DE firstly increases with time, and then, after a long-time evolution, it saturates. When $\alpha$ changes from 1 to 0 , the $X X$ ladder model becomes less ergodic, and in the limit $\alpha=0$, it degenerates into the integrable $X Y$ spin chain and fails to thermalize. We also note that the saturation or oscillation of EE can characterize the integrability of a system [37].

It is worthwhile to emphasize that the choice of initial state in Figs. 13(a) and 13(b) is different from the initial state in the main text shown in Fig. 5. The reason behind the present choice of initial state is straightforward: The dynamical signatures of thermalization, MBL, nonergodicity or AL can be revealed by quench dynamics. If we chose the initial state in Fig. 5, the dynamics of DE for $H_{3}$ with $\alpha \sim 0$ can be regarded as the dynamics of $X Y$ model with initial state $|\downarrow \downarrow \ldots \downarrow\rangle$ (or $|\uparrow \uparrow \ldots \uparrow\rangle)$, which are the eigenstates of the $X Y$ model, and consequently, the dynamics is trivial for studying the phenomena. Indeed, the inset of Fig. 13(b) present a comparison between the DE dynamics with the initial state in the inset of Fig. 13(a) (green triangle points) and in Fig. 5 (darker green square points) when $\alpha=0.1$. With the initial state in Fig. 5, one can see that the dynamical behavior of $\mathrm{DE}$ is less distinguishable. 
Moreover, with the initial state in Fig. 5, we plot the dynamics of DE with $\alpha=0$ and different quasiperiodic strength $W=0$ and 5. Although the value of DE is quite small, the oscillation is still dramatic with $W=0$, which is further suppressed in the presence of a stronger quasiperiodic potential $W=5$.

For the $X X$-ladder model, taking the conservation of total magnetization $S^{z}=S_{1}^{z}+S_{2}^{z}\left(S_{j}^{z}=\sum_{i=1}^{M} \sigma_{j, i}^{z}\right.$ with the $M$ as the length of the spin ladder and $j=1,2$ ) into consideration, we recognize that both of the initial state in the inset of Figs. 13(a) and 5 belong to the $S^{z}=0$ sector. In Fig. 14, we present the results of DE dynamics with the same case of Figs. 13(a) and 13(b) but considering another initial state in the $S^{z}=2$ sector, as shown in the inset of Fig. 14(a). Similar behaviors of DE can also be observed in this sector. Hence, the characterization of AL, MBL, nonergodicity, and thermalization via DE dynamics does not necessarily require that the initial states are in one particular $S^{z}$ sector.
[1] R. Nandkishore and D. A. Huse, Annu. Rev. Condens. Matter Phys. 6, 15 (2015).

[2] E. Altman, Nat. Phys. 14, 979 (2018).

[3] D. A. Abanin, E. Altman, I. Bloch, and M. Serbyn, Rev. Mod. Phys. 91, 021001 (2019).

[4] D. A. Abanin and Z. Papić, Ann. Phys. (Berlin) 529, 1700169 (2017).

[5] M. Schreiber, S. S. Hodgman, P. Bordia, H. P. Lüschen, M. H. Fischer, R. Vosk, E. Altman, U. Schneider, and I. Bloch, Science 349, 842 (2015).

[6] J.-y. Choi, S. Hild, J. Zeiher, P. Schauß, A. Rubio-Abadal, T. Yefsah, V. Khemani, D. A. Huse, I. Bloch, and C. Gross, Science 352, 1547 (2016).

[7] H. P. Lüschen, P. Bordia, S. S. Hodgman, M. Schreiber, S. Sarkar, A. J. Daley, M. H. Fischer, E. Altman, I. Bloch, and U. Schneider, Phys. Rev. X 7, 011034 (2017).

[8] T. Kohlert, S. Scherg, X. Li, H. P. Lüschen, S. Das Sarma, I. Bloch, and M. Aidelsburger, Phys. Rev. Lett. 122, 170403 (2019).

[9] H. P. Lüschen, P. Bordia, S. Scherg, F. Alet, E. Altman, U. Schneider, and I. Bloch, Phys. Rev. Lett. 119, 260401 (2017).

[10] J. Smith, A. Lee, P. Richerme, B. Neyenhuis, P. W. Hess, P. Hauke, M. Heyl, D. A. Huse, and C. Monroe, Nat. Phys. 12, 907 (2016).

[11] K. Xu, J.-J. Chen, Y. Zeng, Y.-R. Zhang, C. Song, W. Liu, Q. Guo, P. Zhang, D. Xu, H. Deng, K. Huang, H. Wang, X. Zhu, D. Zheng, and H. Fan, Phys. Rev. Lett. 120, 050507 (2018).

[12] P. Roushan, C. Neill, J. Tangpanitanon, V. M. Bastidas, A. Megrant, R. Barends, Y. Chen, Z. Chen, B. Chiaro, A. Dunsworth, A. Fowler, B. Foxen, M. Giustina, E. Jeffrey, J. Kelly, E. Lucero, J. Mutus, M. Neeley, C. Quintana, D. Sank, A. Vainsencher, J. Wenner, T. White, H. Neven, D. G. Angelakis, and J. Martinis, Science 358, 1175 (2017).

[13] Q. Guo, C. Cheng, Z.-H. Sun, Z. Song, H. Li, Z. Wang, W. Ren, H. Dong, D. Zheng, Y.-R. Zhang, R. Mondaini, H. Fan, and H. Wang, arXiv: 1912.02818

[14] B. Chiaro, C. Neill, A. Bohrdt, M. Filippone, F. Arute, K. Arya, R. Babbush, D. Bacon, J. Bardin, R. Barends, S. Boixo, D. Buell, B. Burkett, Y. Chen, Z. Chen, R. Collins, A. Dunsworth, E. Farhi, A. Fowler, B. Foxen, C. Gidney, M. Giustina, M. Harrigan, T. Huang, S. Isakov, E. Jeffrey, Z. Jiang, D. Kafri, K. Kechedzhi, J. Kelly, P. Klimov, A. Korotkov, F. Kostritsa, D. Landhuis, E. Lucero, J. McClean, X. Mi, A. Megrant, M. Mohseni, J. Mutus, M. McEwen, O. Naaman, M. Neeley, M. Niu, A. Petukhov, C. Quintana, N. Rubin, D. Sank, K. Satzinger, A. Vainsencher, T. White, Z. Yao, P. Yeh,
A. Zalcman, V. Smelyanskiy, H. Neven, S. Gopalakrishnan, D. Abanin, M. Knap, J. Martinis, and P. Roushan, arXiv: 1910.06024.

[15] J. H. Bardarson, F. Pollmann, and J. E. Moore, Phys. Rev. Lett. 109, 017202 (2012)

[16] M. Serbyn, Z. Papić, and D. A. Abanin, Phys. Rev. Lett. 110, 260601 (2013)

[17] D. J. Luitz, N. Laflorencie, and F. Alet, Phys. Rev. B 93, 060201(R) (2016).

[18] M. Pino, Phys. Rev. B 90, 174204 (2014).

[19] D.-L. Deng, X. Li, J. H. Pixley, Y.-L. Wu, and S. Das Sarma, Phys. Rev. B 95, 024202 (2017).

[20] P. Sierant, D. Delande, and J. Zakrzewski, Phys. Rev. A 95 , 021601(R) (2017).

[21] A. Safavi-Naini, M. L. Wall, O. L. Acevedo, A. M. Rey, and R. M. Nandkishore, Phys. Rev. A 99, 033610 (2019).

[22] K. Macieszczak, E. Levi, T. Macrì, I. Lesanovsky, and J. P. Garrahan, Phys. Rev. A 99, 052354 (2019).

[23] T. Chanda, P. Sierant, and J. Zakrzewski, arXiv:1908.06524.

[24] E. V. H. Doggen, F. Schindler, K. S. Tikhonov, A. D. Mirlin, T. Neupert, D. G. Polyakov, and I. V. Gornyi, Phys. Rev. B 98, 174202 (2018).

[25] E. V. H. Doggen and A. D. Mirlin, Phys. Rev. B 100, 104203 (2019).

[26] P. Sierant and J. Zakrzewski, New J. Phys. 20, 043032 (2018).

[27] X. Wei, C. Cheng, G. Xianlong, and R. Mondaini, Phys. Rev. B 99, 165137 (2019).

[28] P. Naldesi, E. Ercolessi, and T. Roscilde, SciPost Phys. 1, 101 (2016).

[29] R. Singh, J. H. Bardarson, and F. Pollmann, New J. Phys. 18, 023046 (2016).

[30] E. J. Torres-Herrera and L. F. Santos, Ann. Phys. (Berlin) 529 , 1600284 (2017).

[31] J. A. Kjäll, J. H. Bardarson, and F. Pollmann, Phys. Rev. Lett. 113, 107204 (2014).

[32] D. J. Luitz, N. Laflorencie, and F. Alet, Phys. Rev. B 91, 081103(R) (2015).

[33] A. Pal and D. A. Huse, Phys. Rev. B 82, 174411 (2010).

[34] V. Khemani, D. N. Sheng, and D. A. Huse, Phys. Rev. Lett. 119, 075702 (2017).

[35] V. Khemani, S. P. Lim, D. N. Sheng, and D. A. Huse, Phys. Rev. X 7, 021013 (2017).

[36] M. Lee, T. R. Look, S. P. Lim, and D. N. Sheng, Phys. Rev. B 96, 075146 (2017).

[37] Y. O. Nakagawa, M. Watanabe, H. Fujita, and S. Sugiura, Nat. Commun. 9, 1635 (2018) 
[38] J. Gray, S. Bose, and A. Bayat, Phys. Rev. B 97, 201105(R) (2018).

[39] S.-X. Zhang and H. Yao, Phys. Rev. Lett. 121, 206601 (2018).

[40] A. C. Potter, R. Vasseur, and S. A. Parameswaran, Phys. Rev. X 5, 031033 (2015).

[41] R. Vosk, D. A. Huse, and E. Altman, Phys. Rev. X 5, 031032 (2015).

[42] P. T. Dumitrescu, R. Vasseur, and A. C. Potter, Phys. Rev. Lett. 119, 110604 (2017).

[43] T. Baumgratz, M. Cramer, and M. B. Plenio, Phys. Rev. Lett. 113, 140401 (2014)

[44] X. Yuan, H. Zhou, Z. Cao, and X. Ma, Phys. Rev. A 92, 022124 (2015).

[45] C. B. Dağ and L.-M. Duan, Phys. Rev. A 99, 052322 (2019).

[46] T. Brydges, A. Elben, P. Jurcevic, B. Vermersch, C. Maier, B. P. Lanyon, P. Zoller, R. Blatt, and C. F. Roos, Science 364, 260 (2019).

[47] Y. Ye, Z.-Y. Ge, Y. Wu, S. Wang, M. Gong, Y.-R. Zhang, Q. Zhu, R. Yang, S. Li, F. Liang, J. Lin, Y. Xu, C. Guo, L. Sun, C. Cheng, N. Ma, Z. Y. Meng, H. Deng, H. Rong, C.-Y. Lu, C.-Z. Peng, H. Fan, X. Zhu, and J.-W. Pan, Phys. Rev. Lett. 123, 050502 (2019).

[48] C. Radhakrishnan, M. Parthasarathy, S. Jambulingam, and T. Byrnes, Phys. Rev. Lett. 116, 150504 (2016).

[49] S.-P. Li and Z.-H. Sun, Phys. Rev. A 98, 022317 (2018).

[50] D. N. Page, Phys. Rev. Lett. 71, 1291 (1993).

[51] V. Zelevinsky, B. Brown, N. Frazier, and M. Horoi, Phys. Rep. 276, 85 (1996).

[52] E. J. Torres-Herrera, J. Karp, M. Távora, and L. F. Santos, Entropy 18, 359 (2016).

[53] M. T. Batchelor, X. W. Guan, N. Oelkers, and Z. Tsuboi, Adv. Phys. 56, 465 (2007)
[54] S. P. Strong and A. J. Millis, Phys. Rev. Lett. 69, 2419 (1992).

[55] T. Barnes, E. Dagotto, J. Riera, and E. S. Swanson, Phys. Rev. B 47, 3196 (1993).

[56] M. Žnidarič, Phys. Rev. Lett. 110, 070602 (2013).

[57] A. Tribedi and I. Bose, Phys. Rev. A 79, 012331 (2009).

[58] E. Baygan, S. P. Lim, and D. N. Sheng, Phys. Rev. B 92, 195153 (2015).

[59] D. Wiater and J. Zakrzewski, Phys. Rev. B 98, 094202 (2018).

[60] N. Y. Yao, C. R. Laumann, J. I. Cirac, M. D. Lukin, and J. E. Moore, Phys. Rev. Lett. 117, 240601 (2016).

[61] P. Richerme, Z.-X. Gong, A. Lee, C. Senko, J. Smith, M. FossFeig, S. Michalakis, A. V. Gorshkov, and C. Monroe, Nature 511, 198 (2014).

[62] M. Azzouz, L. Chen, and S. Moukouri, Phys. Rev. B 50, 6233 (1994).

[63] E. Lieb, T. Schultz, and D. Mattis, Ann. Phys. 16, 407 (1961).

[64] A. B. Harris, J. Phys. C: Solid State Phys. 7, 1671 (1974).

[65] J. M. Luck, Europhys. Lett. 24, 359 (1993).

[66] A. Chandran, C. R. Laumann, and V. Oganesyan, arXiv: 1509.04285 .

[67] D. Barredo, S. de Léséleuc, V. Lienhard, T. Lahaye, and A. Browaeys, Science 354, 1021 (2016).

[68] M. Schulz, C. A. Hooley, R. Moessner, and F. Pollmann, Phys. Rev. Lett. 122, 040606 (2019).

[69] E. van Nieuwenburg, Y. Baum, and G. Refael, Proc. Natl. Acad. Sci. USA 116, 9269 (2019).

[70] B. Sciolla and G. Biroli, J. Stat. Mech. (2011) P11003.

[71] S. Pappalardi, A. Russomanno, B. Žunkovič, F. Iemini, A. Silva, and R. Fazio, Phys. Rev. B 98, 134303 (2018). 